\title{
The Cognition of Curatorial Strategies for Interpreting Localism
}

\author{
Yu-Ju Lin, Jun-Liang Chen, Po-Hsien Lin \\ National Taiwan University of Arts, New Taipei City, Taiwan
}

\begin{abstract}
Many unique traditional industries in Taiwan have declined over time. How to revive these fading craft cultures and retain the diversity of Taiwan's cultural assets is a critical research topic. Thus, when preserving valuable cultural resources through curatorial exhibitions is considered a feasible measure, approaches to curating effective exhibitions that elicit emotional responses from visitors is a crucial issue. This article explores the emotional effect of the curation process as well as the visitor experiences by investigating the "Lost Memories Redux-The Search for the 100 Lost Industries” exhibition as a case study. Through this case study, a curatorial model was established to explain how to effectively elicit emotional responses from exhibition visitors. Through semi-structured in-depth interviews and a literature review, this article presents an analysis of the curatorial practice model. Subsequently, the results of a questionnaire survey are analysed to explore the emotional differences between the curator and the visitors regarding their exhibition experience. The results show that the proposed curatorial model can effectively elicit emotional responses of the visitors and identify their preferences. Through effective curatorial design, the core theme of an exhibition can be transmitted to visitors, thereby raising their awareness of cultural conservation and reflection. Thus, the proposed curatorial model can enhance the visitor's acknowledgement according to their emotional responses to the exhibition. The results may serve as a reference for future studies.
\end{abstract}

Keywords: curation, localism, cultural conservation, cultural reflection

\section{Introduction}

Exhibition design is an on-going process. Through the process of cultural participation, visitors internalize cultural identity targets and values, with a particular emphasis on forming a mutual cultural identity by developing a sense of belonging to a community. In contemporary curatorial methods, how curators present the features of an exhibition has become a critical topic in curating works for an exhibition. However, determining how to interpret an exhibition theme according to how items are displayed, how storylines are arranged, and how spatial layouts are combined to successfully transmit a message to visitors in full, is a challenge for curators. Curators must possess a complete understanding of exhibition content what to say as well as when and how to say it. Burcaw (1987), Carroll (2004), Liu (2003), Fu (2004), Hsu (2009), and Hsu and Lin (2011) have contended that from planning to executing an exhibition, as well as through the process of formulating a curatorial design and communicating a central theme, determining whether curators and visitors relate to an exhibition theme in the same way and share the same values is crucial.

Yu-Ju Lin, Ph.D. candidate, Graduate School of Creative Industry Design, National Taiwan University of Arts. Jun-Liang Chen, Ph.D. candidate, Graduate School of Creative Industry Design, National Taiwan University of Arts. Po-Hsien Lin, Ph.D., professor, Graduate School of Creative Industry Design, National Taiwan University of Arts. 
Baxter (1998), Crilly, Moultrie, and Clarkson (2004), and Norman (2005) have reported that when abstract design concepts are transformed into tangible products, because curators and audiences have different experience, a certain degree of difference exists between the original concept and the finished work. In the process of curating works for an exhibition on localism, determining how to assist curators in accurately realizing the concepts of cultural conservation and reflection while simultaneously retaining the original concept implications and catering to the perceptions and preferences of visitors have become primary topics that curators must consider carefully when devising a curation strategy. Accordingly, this article examines the "Lost Memories Redux-The Search for the 100 Lost Industries" exhibition as a research target and explores the differences between curators and visitors regarding their experiential perceptions. Clarifying the difference between visitors and curators could help visitors understand the theme of an exhibition, which is something that curators must pay attention to following the increasingly complex spiritual and cultural needs of exhibition visitors. Belcher (1991) claimed that media communicate information. The value of an exhibition equals the information it conveys. However, visitors attend exhibitions to experience authentic works, acquire new knowledge, and attain spiritual and sensory enrichment and satisfaction. Curators must organise complex and diverse information rapidly and effectively. Through adopting systematic and organised transmission methods, curators can provide real-time feedback and resources to visitors to help them apply the various types of information provided in order to further understand the exhibition. Thus, developing high-quality information not only facilitates effective information transmission, but also arouses visitors' interests in an exhibition, thereby, influencing their behaviours and learning.

\section{Literature Review}

\section{Visitor Experiential Perceptions}

When attending an exhibition, individual visitors have different experiences according to the interaction between their moods and the event (Mcluhan, 2000). During the experiential process, the senses, cognitions, minds, and behaviours of visitors continually interact with the environment. Through such interactions, visitors can develop perceptions and gain experience (Kao, 2002). Falk and Dierking (1992) proposed an interactive experience model and argued that such experiences are not completely passive for visitors. Visitors each have unique experiences that form through the interaction of personal, social, and physical contexts. Finally, after visiting an exhibition, visitors reflect on their experiences based on their memories, which influences their expectations for and experiences of the next exhibition they attend. The considerations involved in designing a contemporary exhibition are focused on creating an optimal scenario that promotes visitor learning. The content of an exhibition must consistently reflect diversity. A critical consideration for visitors is to build confidence through observation (Oppenheimer, 1990). The key concepts for curators, who effectively act as the "authors" when designing an exhibition, are the operation, control, and rehearsal of spatial considerations. Building an exhibition atmosphere goes beyond utilising the physical characteristics of an exhibition space. Such atmosphere is created through contrasting works, cultural relics, and the exhibit spaces. Curators have a role that is similar to that of a director, in that they provide a context for actors (artists or works), which is the first layer of rhetoric when communicating with visitors (Lin, 2014). The intervention and control enables visitors to construct diverse thoughts and understandings regarding the theme of an exhibition. Thus, in the process of designing an exhibition and deciding on the content, curators provide stimuli for visitors through transmitting information and concepts that comprise the external factors of exhibitions. In any exhibition, curators must 
consider visitors' emotional perceptions, create bonds through communication, form relationships with visitors, and thereby, transmit sensory exhibition curations.

\section{Exploration of Emotional Effect}

Jordan (2000) indicated that consumers have three layers of needs related to products they consume: functionality, usability, and pleasure. Functionality represents the intended use that a product is created for, through which people can utilise the product's basic functions to complete certain tasks. Maslow (1943) proposed the hierarchy of needs (five basic needs) to define user needs theory regarding product characteristics including functionality, usability, and pleasure. Norman (2004) suggested that in addition to functionality, products are embedded with another critical element: emotion. Through the classic affective-behavioural-cognitive model of attitude, Norman developed three levels of emotional design: visceral (product appearance and texture), behavioural (product functions), and reflective (personal perceptions and thoughts) levels. He also indicated the importance of emotions to decision-making in daily life. In response to various levels of design principles for the current design and service industries that increasingly emphasise customisation and interaction, emotional design becomes central to the future development of industries. Lin (2007) improved the emotional design structure and combined it with Norman's concept. He divided the factors requiring consideration when designing cultural and creative products into three levels of design attributes: (a) the outer tangible level, including colours, textures, shapes, and patterns; (b) the middle or behavioural level, including functionality, operability, and usability; and (c) the inner or intangible level, which is motivated by story, emotion, and cultural connotations. Figure 1 shows a comparison of the hierarchy of needs proposed by Maslow (1943) with the hierarchy of user needs proposed by Jordan (2000). In addition, the consideration factors regarding product design proposed by Norman (2004) and Lin (2007) are included in the comparison (Chu, 2011). In addition to considering techniques and devising themes, curation should be focused on forming interactions with the psychological or emotional characteristics of visitors. This article explores how the curator realized the core themes of an exhibition through curatorial planning and implementation. In addition to elucidating the curation process, this article examines how the curator converted abstract concepts into tangible display elements that promote learning and generate interest in an exhibition, which are crucial strategies and methods that can be applied in contemporary curatorial design.

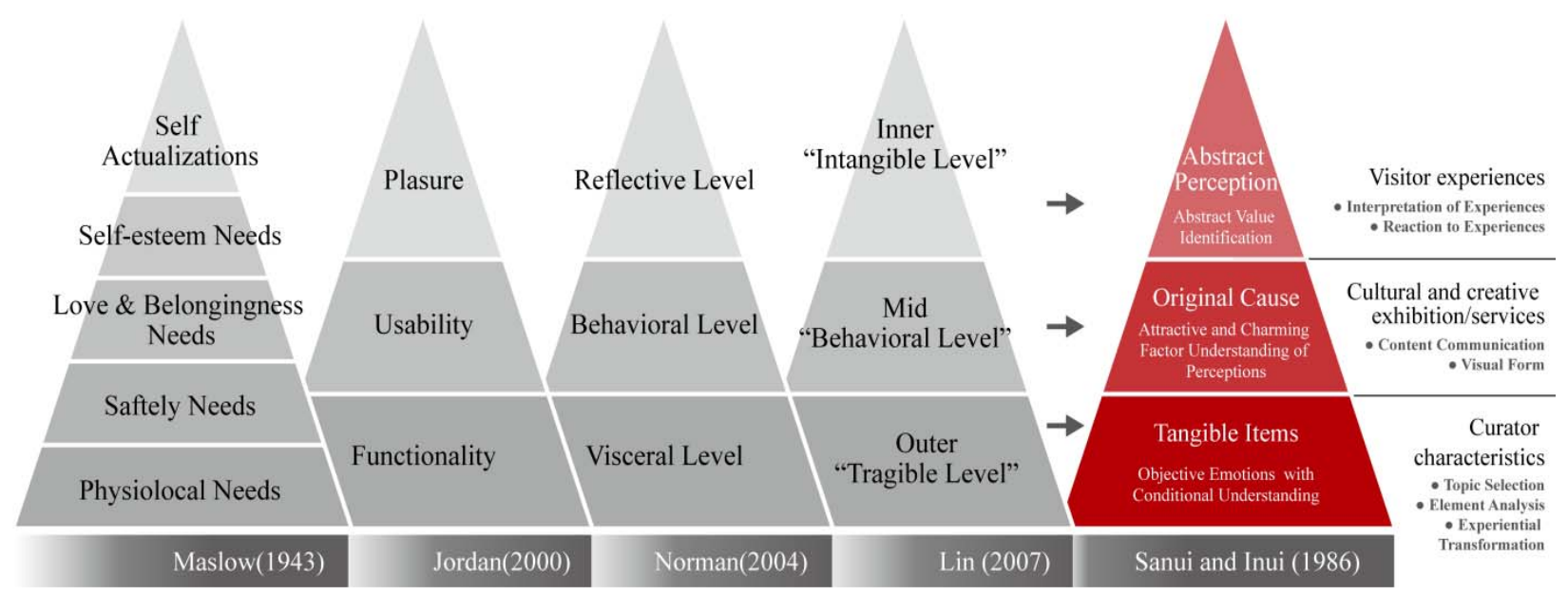

Figure 1. Comparison of application of hierarchy theory in curatorial practice models. 


\section{The Curator's Viewpoint and Strategy}

Hsu (2009) proposed an educational viewpoint that is suitable for curators, who are responsible for interpreting the combination of arts and institutions (i.e., final exhibits, concepts, or products), enabling artists and firms to view their works and products from different perspectives. Artists can understand their works from a commercial perspective and be inspired by new directions in creativity, whereas institutions can integrate creative designs into mass-produced products to generate innovation and market demand. Thus, when people create or attend exhibitions, these intrinsic and extrinsic factors should be considered in order to facilitate creating effective works and rendering their observation meaningful. The curator's meticulous planning and in-depth understanding of works are essential in making decisions regarding exhibition themes. Curators attempt to employ research and exhibition themes to make exhibitions meaningful and create an environment that visitors can share. These efforts are the goal of curatorial design. A curation model primarily starts with determining the theme followed by searching for art works that can be connected with the theme. Obtaining diverse works that represent the theme can elicit certain questions from visitors while observing the exhibition (Fu, 2004). Hosting an exhibition requires a thorough planning process. Although the execution process may differ according to the theme or characteristics of an exhibition, the development process of curatorial planning and scheduling can be divided into four stages.

1. Introduction: An organiser communicates and negotiates with relevant organisations to ensure that works for an exhibition theme are obtainable.

2. Preparation: Curation conceptions are transformed from abstract concepts into physical objects. Artists are contacted and negotiated with in the process of curating works. The exhibition venue and exhibit objects are then confirmed, prepared, and arranged. Event promotion materials are created that meet the needs and regulations of the exhibition implementation process.

3. Execution: Activities related to exhibition opening, media advertising, frontline and service staff, education training, guided tours, and post exhibition dismantling are undertaken.

4. Dismantling: Disposing of exhibitions, dismantling of scaffolding, and returning of borrowed items are conducted post exhibition. The outcome and effectiveness of the exhibition is evaluated to publish a work report and perform data archiving. The exhibition execution schedule can be used as a guideline for future curators.

This article explores how the curator transformed abstract content based on the theme of localism into physical exhibition elements through the curation process according to a planned curation schedule, as well as how they embed such elements into an exhibition to express and promote the core theme of cultural conservation and reflection in the exhibition.

\section{Methods}

Exhibitions entailing humanistic representation lack resources and have seldom been studied. When the exhibition is hosted by curators who are not familiar with the theme, the curators need to think outside of conventional frameworks for designing general exhibitions, and they may need to familiarise themselves with the entire design of project operations, as well as seek new spatial meaning for the field. In the first part of this study, semi-structured in-depth interviews were conducted to explore the views of curators. The curator for the case exhibition focused on graphic design and creative products in his early careers. Recently, the curator had participated in several events and won numerous international awards in the field. To derive critical information from the interview data, we extracted and condensed valuable internal knowledge of the exhibition and 
organised the curator's experiences and operational guides to formulate specific curatorial planning steps to provide a reference and planning directions for the curators of future exhibitions. The second part involved designing a questionnaire. Following the consumption emotions set developed by Richins (1997), 46 items were combined with the five items related to the curator's emphasis on exhibition essence established by this article. Because this article assesses the positive emotions and behavioural intentions of visitors, negative emotional responses were removed. To reduce the items, repeated or similar emotional adjectives and those irrelevant to the current research topic were eliminated. Experts in related fields were invited to screen appropriate lexicons and list the emotional experience adjectives. Fourteen items were measured using a 5-point scale. The first section comprised nine items for evaluating of the works (realism, attractiveness, liveliness, value, craftsmanship, traditional content, content focus, emotion, and representativeness of local industry artists and craftsmen). The second section comprised perceptions from the exhibition and examined five items (representativeness of local industry artists' and craftsmen's spirit, benevolence, affection, joy, and pricelessness). Each item was evaluated from 1 ("Strongly disagree”) to 5 (“Strongly agree”). The response data were analysed using the multidimensional scaling in Statistical Package for Social Sciences (SPSS). Multivariate regression was used in the first stage of the analysis to identify which visitor preferences and satisfaction with the image content of the exhibited works differed significantly $(p<0.001)$. An independent samples $t$-test was then conducted to examine whether the curator and the visitors' evaluations and perceptions differed significantly $(p<0.05)$ (see Figure 2).

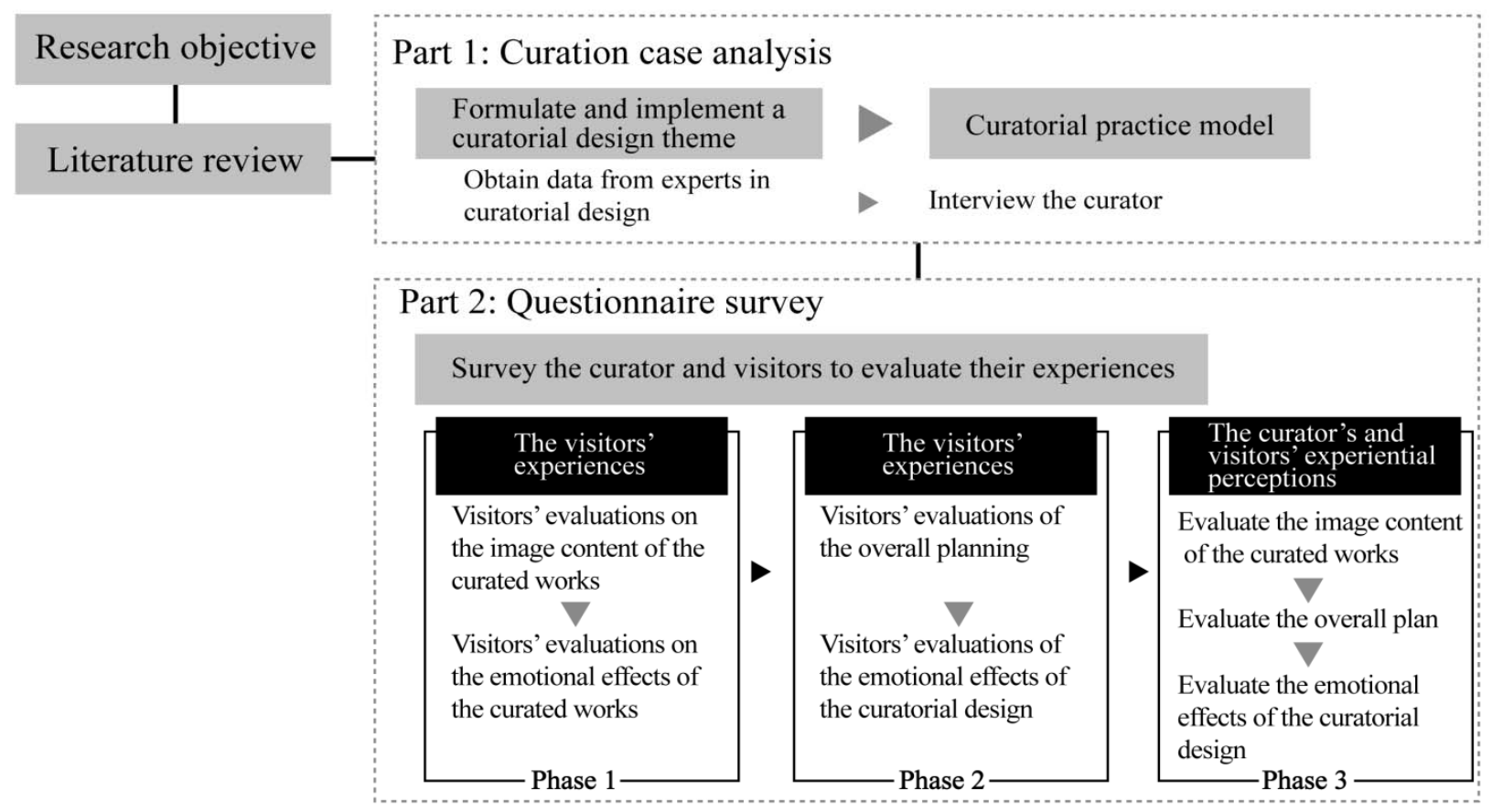

Figure 2. Research framework and flowchart.

\section{Tangible Practice of Curating Design}

\section{Formation of Curatorial Planning Concepts}

The curatorial planning concept of this exhibition was based on the curator's inspiration, and focused on demonstrating the local connotations and meanings based on the theme of searching for localism. The curator 
explained that the artists featured in the exhibition employed their skills, which they had developed over their lives, to demonstrate their hand-made art works. Thus, in this exhibition, instead of emphasising the effort and humbleness of these artists and craftsmen, the curator focused on their persistence and commitment to represent the 100 fading traditional industries in this transitioning era. Through the expression of strong but simple emotions, the exhibition displayed the local beauty of Taiwan and demonstrated respect to the guardians and transmitters of these beautiful folk cultures. To indicate the curatorial theme of emphasising traditional arts and crafts, the curator developed a simple and direct slogan for the exhibition: "See You Tomorrow," which also elicits visitors' emotion. They, thus, adopted "Focusing on local Taiwanese cultures to manifest the spirit of craftsmen" as the exhibition theme. The craftsmen's lives and cultures were displayed in detail (see Figure 3). Through benevolent and affectionate practice of joyful identification with the land of Taiwan, the craftsmen and artists enjoyed increasing popularity and attained opportunities of being continuously reported by media or receiving event invitations, thereby generating extended and lasting effectiveness of the exhibition.
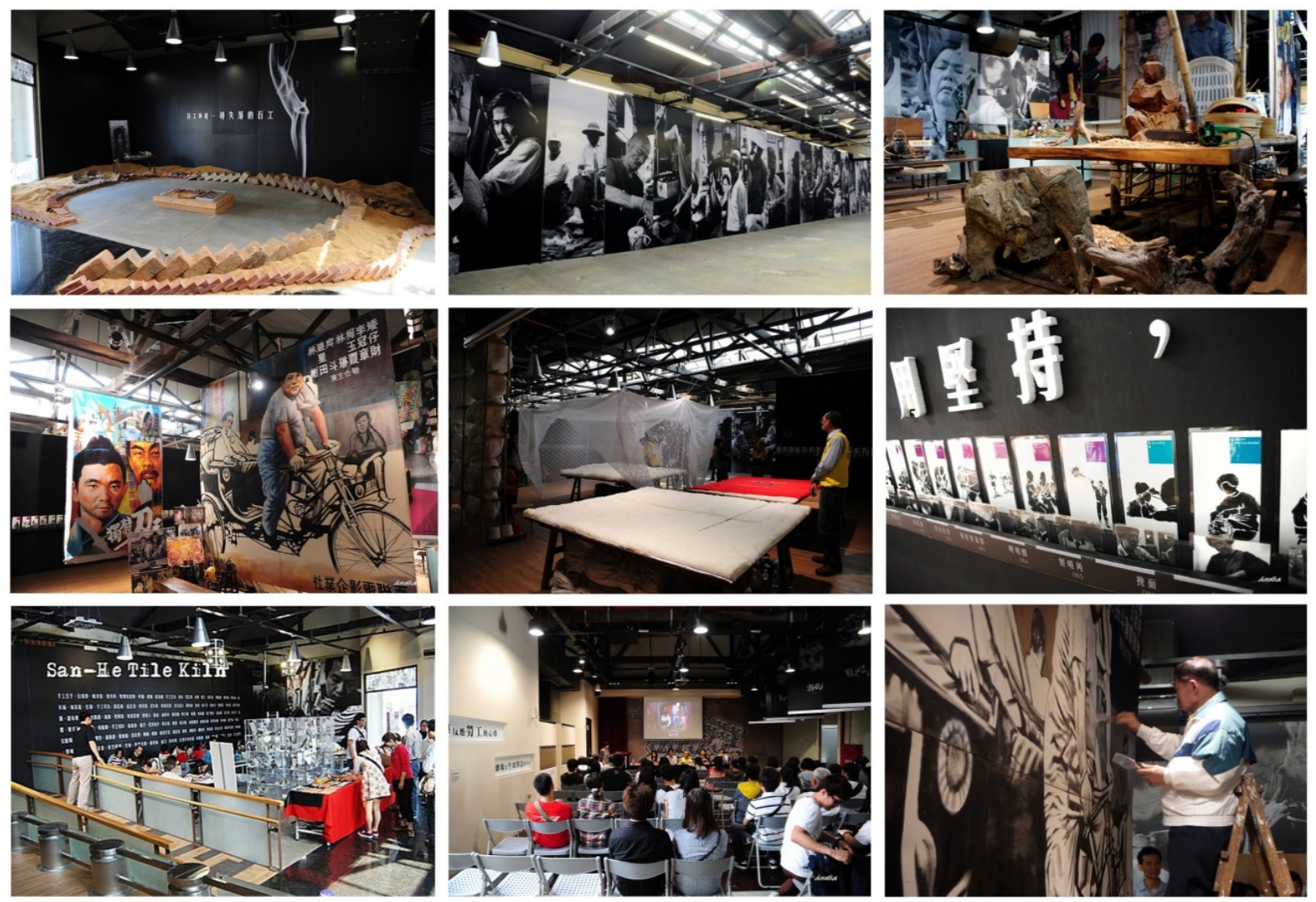

Figure 3. Scenes of the exhibition area.

\section{Implementation of Curatorial Planning Concepts}

The curator's curatorial planning concepts were aimed at creating an exhibition that reflected the cultures of the traditional industries the artists and craftsmen worked in and ensured that they were fully aware of the curator's interpretation of the exhibition content. Thus, the curator's expectations for the exhibits and the spatial planning of the exhibition were. After organising the interview data, we summarised four steps in curating design as follows. 
Establishing an exhibition theme. This step involves two tasks, i.e., developing the core theme and establishing a thematic connection. Developing the core theme involves screening and planning all of the exhibition content through meaningful and organized methods according to the connotations of the traditional industries and cultures of the artists and craftsmen. Establishing the thematic connection involves exploring and interpreting the thoughts of artists and craftsmen in the traditional industries and cultures to highlight their persistence in their profession and to emphasise their refined skills and techniques.

Determining the exhibition structure. This step comprises two tasks, i.e., administrative management and implementing engineering technologies. Administrative management involves managing relevant affairs, integrating art, and general administration. In this exhibition, works were curated from artists and craftsmen and then transported to the exhibition venue. Thus, the estimated budget accounted for packaging, transport, insurance, and damage of the exhibits. Implementing engineering technologies refers to the integration between interfacial design employed in the presentation of the exhibit and technology engineering of the exhibition form to meet the requirements and adjustments of the exhibition project. Experts from relevant fields were hired to adjust the details according to the characteristics of the works, including how the works were arranged and setting up the lighting. With the assistance of the experts, the overall exhibition projected a sense of harmony.

Curating the exhibition works. To ensure that the artists and craftsmen understood of the curator's vision for the exhibition, this step involves sampling works and creating platforms for the exhibits. The sampling of works involved the curatorusing his personal experience and visiting various places in Taiwan to search for data, contacting artists and craftsmen, and choosing the sampling. The artists and craftsmen were not from relatively unknown or archaic industries. Most of the industries included in the exhibition were either established during the Japanese colonial period or strongly influenced by that period. Creating platforms for the exhibits required the curator to determine how the exhibited works would be presented. The curated works were large. For instance, the wall photography images on both sides corridor of the entrance were arranged by seamlessly connecting a series of printed photographs to create a clustering effect, contributing to the beauty of the exhibition because of the large scale of the work. This type of presentation reinforced the beauty of the works displayed at the exhibition.

Designing the venue layout. This step primarily involves two work items aimed at planning and designing the exhibition spaces, i.e., planning the exhibition area and constructing the atmosphere. Planning the exhibition area involved evaluating the exhibition space, such as area size, floor layout, perspective drawing, and distribution of power sockets and electricity access, before planning visitor flow management. The curator used environmentally friendly concepts combined with various common elements in life to plan the exhibition area. The emphasis and visual focus of the exhibition area were designed to enable the visitors to experience the characteristics of the curated works. Constructing the atmosphere involved arranging the works and adjusting lighting. Such adjustments imparted specific spatial aesthetic atmospheres and perceptions of the various contexts the exhibits were involved in. After analysing traditional Taiwanese crafts, the curator decided to construct an atmosphere of early life cultures under the influence of Japanese rule by using colour, text, and devices.

According to the in-depth interview data, we deduced the curatorial practice model employed by the curator. As shown in Figure 4, the curatorial practice model comprises three stages. The first stage is project conception. The main content demonstrated the innovative thinking in design. Creating exhibitions through the 
ideas of extended focus and depicting real life can elicit emotional responses from and resonance with visitors and strengthen the artists' and craftsmen's persistence for Taiwanese localism. The second stage involved ensuring that the exhibitions require people foremost action practice. In other words, providing interpretations of the various knowledge and new aesthetic experiences to visitors can lead them into the scenarios the curator constructed and enable them to "communicate" with the works, thereby, bridging the visitors' impressions of and emotional responses to the works with the curator's. Moreover, in determining the exhibit presentation framework, the curator employed the approaches and attitude that emphasized text and lexicon and pursuit for perfection; through this approach, the curation process was successfully implemented and promoted. Subsequently, in curating the works, the curator applied the creative techniques for reinforcing unique values and using the colour, image, and texture elements of the works to emphasise the core theme. In designing the venue layout, the curator planned the layout of the exhibition area and employed various atmospheres and ambience that changed the exhibition space. To maintain the venue quality and atmosphere, the curator had to design the exhibition venue through spatial partitions and connections, and aesthetic management to manipulate lighting and adjustments to the detailed characteristics of the works. The third stage was project review. A review meeting was convened to determine the strengths and limitations of the exhibition as well as measures for how the exhibition could be improved. Following the exhibition, the curator evaluated the visitors' perceptions of the works as well as their views on how the service quality could be improved in order to identify detailed sections that should be considered in the future. According to the interview content, this article systematically summarized a curatorial practice model that can serve as a reference guideline for training and teaching future curators.

\begin{tabular}{|c|c|c|c|c|}
\hline & Phase & \multicolumn{2}{|c|}{ Task content } & Interpretation of curatorial contents \\
\hline $\begin{array}{l}\text { Exhibition } \\
\text { introduction }\end{array}$ & $\begin{array}{l}\text { Project } \\
\text { conception }\end{array}$ & \multicolumn{2}{|c|}{ Innovative thinking } & $\begin{array}{l}\text { - Magnification of the exhibits (extended focus) } \\
\text { - Profound inspiration (demonstrating real life) }\end{array}$ \\
\hline \multirow{5}{*}{$\begin{array}{l}\text { Exhibition } \\
\text { preparation }\end{array}$} & \multirow{8}{*}{$\begin{array}{l}\text { Project } \\
\text { execution }\end{array}$} & \multirow{2}{*}{ Exhibition theme } & Core theme & \multirow{2}{*}{$\begin{array}{l}\text { - Cultural depth (passing on localism) } \\
\text { - Specific theme (persistence as the principle) }\end{array}$} \\
\hline & & & Thematic connection & \\
\hline & & \multirow{2}{*}{$\begin{array}{l}\text { Exhibition } \\
\text { framework }\end{array}$} & Administrative management & \multirow{2}{*}{$\begin{array}{l}\text { - Procedural smoothness (emphasising text and lexicon) } \\
\text { - Creation practice (pursuing perfection) }\end{array}$} \\
\hline & & & Engineering technologies & \\
\hline & & \multirow{2}{*}{ Collecting exhibit } & Sampling works & \multirow{2}{*}{$\begin{array}{l}\text { - Ability to recognise arts (strengthening unique values) } \\
\text { (flexibly using colour, image and texture elements) }\end{array}$} \\
\hline \multirow{3}{*}{$\begin{array}{l}\text { Exhibition } \\
\text { execution }\end{array}$} & & & Creating exhibits & \\
\hline & & \multirow{2}{*}{ Designing venue } & Planning exhibition area & \multirow{2}{*}{$\begin{array}{l}\text { - Spatial extensibility (partition and reconnection) } \\
\text { - Context sensitivity (aesthetic management) }\end{array}$} \\
\hline & & & Creating atmosphere & \\
\hline \multirow[t]{2}{*}{$\begin{array}{l}\text { Exhibition } \\
\text { dismantling }\end{array}$} & \multirow[t]{2}{*}{$\begin{array}{l}\text { Project } \\
\text { review }\end{array}$} & \multirow{2}{*}{$\begin{array}{l}\text { Review and } \\
\text { improvement }\end{array}$} & Benefit evaluation & \multirow{2}{*}{$\begin{array}{l}\text { - Educative level of event } \\
\text { (visitor's cognitive understanding) } \\
\text { - Ability to integrate mechanisms } \\
\text { (service quality improvement) }\end{array}$} \\
\hline & & & Improvement measures & \\
\hline
\end{tabular}

Figure 4. Curatorial practice model.

\section{Evaluation of Emotional Effect on Visitors' Experiences}

\section{Analysis of Visitor Experience Questionnaire Survey Results}

A total of 149 valid questionnaires were collected, one from the curator and 148 from the visitors (69 men and 80 women). To further explore the visitor's experiences in evaluating the image content of the works, a 
correlation analysis was conducted using the questionnaire data. To determine the affective response for the exhibition (i.e., whether the visitors cherished and valued the artists and craftsmen of traditional industries), we adopted the following eight independent variables: 1. "The photos are emotive"; 2. "The photo composition conveys the craftsmen's persistence"; 3. "The photo content is focused”; 4. "The photos are realistic"; 5. "The photos are lively"; 6. "The photos are valuable"; 7. "The photos are attractive”; and 8. "The photos represent traditional cultures." A multivariate regression analysis was conducted using the independent variables and the dependent variable of the level of emotional effect of the image content of the works on the visitors. Table 1 shows the regression results. The correlations between the independent variables and dependent variable show that five of the eight independent variables were significantly and positively correlated with the dependent variable. The $F$ value of the overall regression model was 27.728 , reaching the level of significance $(p<0.001)$; in other words, the scores of the five independent variables could be used as a basis for predicting the emotional effect. In the regression model, the importance of each dependent variable arranged sequentially according to the standardised regression coefficient value was highest in "The photos represent traditional cultures" ( $\beta=0.456 ; p<0.001$ ), followed by "The photos are lively." The lowest score was observed in "The photos are realistic." From the perspective of the content attributes of the curated works, the works demonstrated observational and realistic imageries. The variables of "The photos represent traditional cultures"; "The photos are lively"; "The photo composition conveys the craftsmen's persistence"; "The photos are emotive"; "The photo content is focused"; and "The photos are realistic" had a stronger emotional effect on the visitors and enabled the visitors to value the artists and craftsmen of the traditional industries more than the variables "The photos are attractive" and "The photos are valuable" did.

Table 1

Evaluation of Work Image Content Based on the Visitors' Experiences

\begin{tabular}{|c|c|c|c|c|c|}
\hline Dependent variable & Independent variable & $B$ & $r$ & $\beta$ & $t$ \\
\hline \multirow{9}{*}{$\begin{array}{l}\text { Increasingly cherish and } \\
\text { value artists and craftsmen } \\
\text { of traditional industries }\end{array}$} & The photos are realistic & 0.079 & $0.504^{* * *}$ & 0.082 & 1.186 \\
\hline & The photos are attractive & -0.092 & $0.468^{* * *}$ & -0.095 & -1.132 \\
\hline & The photos are lively & 0.226 & $0.539^{* * *}$ & 0.238 & $2.687^{* *}$ \\
\hline & The photos are valuable & -0.173 & $0.377^{* * *}$ & -0.164 & $-2.045^{*}$ \\
\hline & $\begin{array}{l}\text { The photo composition conveys the } \\
\text { craftsmen's persistence }\end{array}$ & 0.196 & $0.626^{* * *}$ & 0.179 & $2.294^{*}$ \\
\hline & The photos represent traditional cultures & 0.486 & $0.732^{* * *}$ & 0.456 & $5.405^{* * *}$ \\
\hline & The photo content is focused & 0.090 & $0.537^{* * *}$ & 0.094 & 1.269 \\
\hline & The photos are emotive & 0.102 & $0.511^{* * *}$ & 0.109 & 1.348 \\
\hline & $R=0.784$ & $R^{2}=0.615$ & - & $27.728^{* * *}$ & - \\
\hline
\end{tabular}

Notes. ${ }^{*} p<0.05 ;{ }^{* *} p<0.01$; and ${ }^{* * *} p<0.001$.

The visitors' overall perceptions of their experience at the exhibition were analysed using the following four independent variables: 1. "I feel the local industry craftsmen's persistence in this exhibition"; 2. "I feel benevolence in this exhibition"; 3. "I feel the affectionate feature in this exhibition"; and 4. "I feel that this exhibition is joyful and priceless.” The emotional effect of the visitors' overall experience at the exhibition was used as the dependent variable in the multivariate regression. Table 2 shows the results. The correlation coefficients between each of the four independent variables and the dependent variable were significantly positive. The $F$ value of the overall regression model was 27.135, reaching the level of significance $(p<0.001)$. Thus, these three variables can be used as the basis for predicting the overall emotional effect of the exhibition. 
In the regression model, the importance of each independent variable, arranged according to standardised regression coefficients, is in the descending order of "I feel the local industry craftsmen's persistence in this exhibition"; "I feel the affectionate feature in this exhibition"; and "I feel that this exhibition is joyful and priceless.” The variable "I feel the local industry craftsmen's persistence in this exhibition” reached the level of significance ( $\beta=0.422 ; p<0.001$ ). According to the overall visitors' impression of the exhibition, the overall planning of the exhibition enabled the visitors to perceive the local industry craftsmen's spirit of persistence.

Table 2

Evaluation of the Overall Planning Based on Visitors' Experiences

\begin{tabular}{llrrrr}
\hline Dependent variable & Independent variable & $B$ & $r$ & $\beta$ & $t$ \\
\hline \multirow{2}{*}{$\begin{array}{l}\text { Enjoy the overall } \\
\text { exhibition works and }\end{array}$} & I feel the local craftsmen's persistence in this & 0.473 & $0.579^{* * *}$ & 0.422 & $5.119^{* * *}$ \\
contexts & I feel benevolence in this exhibition & -0.042 & $0.424^{* * *}$ & -0.036 & -0.418 \\
& I feel the affectionate feature in this exhibition & 0.260 & $0.527^{* * *}$ & 0.264 & $2.900^{* *}$ \\
& I feel that this exhibition is joyful and priceless & 0.120 & $0.496^{* * *}$ & 0.128 & 1.329 \\
\hline & $R=0.657$ & $R^{2}=0.432$ & $F=27.135^{* * *}$ \\
\hline Notes. & $* * * 01$ \\
\end{tabular}

\section{Analysis of Exhibition Experience of the Curator and Visitors}

We analysed the curator's and visitors' cognitive attitudes and perceived benefits regarding the exhibition. As shown in Figure 5, many items attained a score higher than 4 points on average. Only three items were lower than 4 points in the factors on evaluating the image content of the works. This shows that the visitors generally recognised and understood the exhibition as a whole. The curator scored 5 points (full score) for the following six items: "The photos are realistic"; "The photos are attractive”; "The photos are lively"; "The photos are valuable"; "The photos represent traditional cultures"; and "The photo content is focused." Thus, the curator was satisfied with the final presentation of the works at the exhibition. The photo works were as if miniatures of the artists' and craftsmen's life during work. The curator claimed that the works focusing on reality easily stimulated the visitors' thoughts and fully expressed the core spirit of the exhibition. Nonetheless, the curator scored 4 points for the image content evaluation items of "The photo composition conveys the craftsmen's persistence" and "The photos are emotive." Although the exhibits were created after the curator had multiple discussions with students from Fu Jen Catholic University, the students encountered unpredictable external elements involving people and spaces while photographing. Thus, the image composition and emotive level did not fully meet the curator's expectations. The scores on these two items revealed that comprehensive preplanning, such as field surveys, consultations, and location searches, should be performed before photographing or filming work records. Comprehensive pre-processing procedures combined with scheduled photographing or filming might conform to the curator's expectation regarding the pertinence and quality of the curated works.

The independent samples $t$-test results indicate that the visitors did not differ significantly among the 14 items $(p>0.05)$. By comparing the means between the curator and visitors, we found that among the eight items exhibiting difference greater than 0.5 points, seven items attained higher scores from the curator than from the visitors. The curator rated lower scores than the visitors did in the "The photo composition conveys the craftsmen's persistence” only. This result merits further exploration. After interviewing the curator, we realised that the curator expected that the works would express the craftsmen's conviction and persistence from 
their eye expression. However, several exhibits were displayed in a full-shot or long-shot layout, which failed to focus on the persistence in the craftsmen's eye expression and thus failed to meet the curator's expectations. The curator required the presentation of the works to be complete, consistent, and refined for the exhibition to be perfect. However, the visitors judged the image compositions intuitively, which contributed to the difference between the visitors and curator regarding the exhibit composition evaluations.

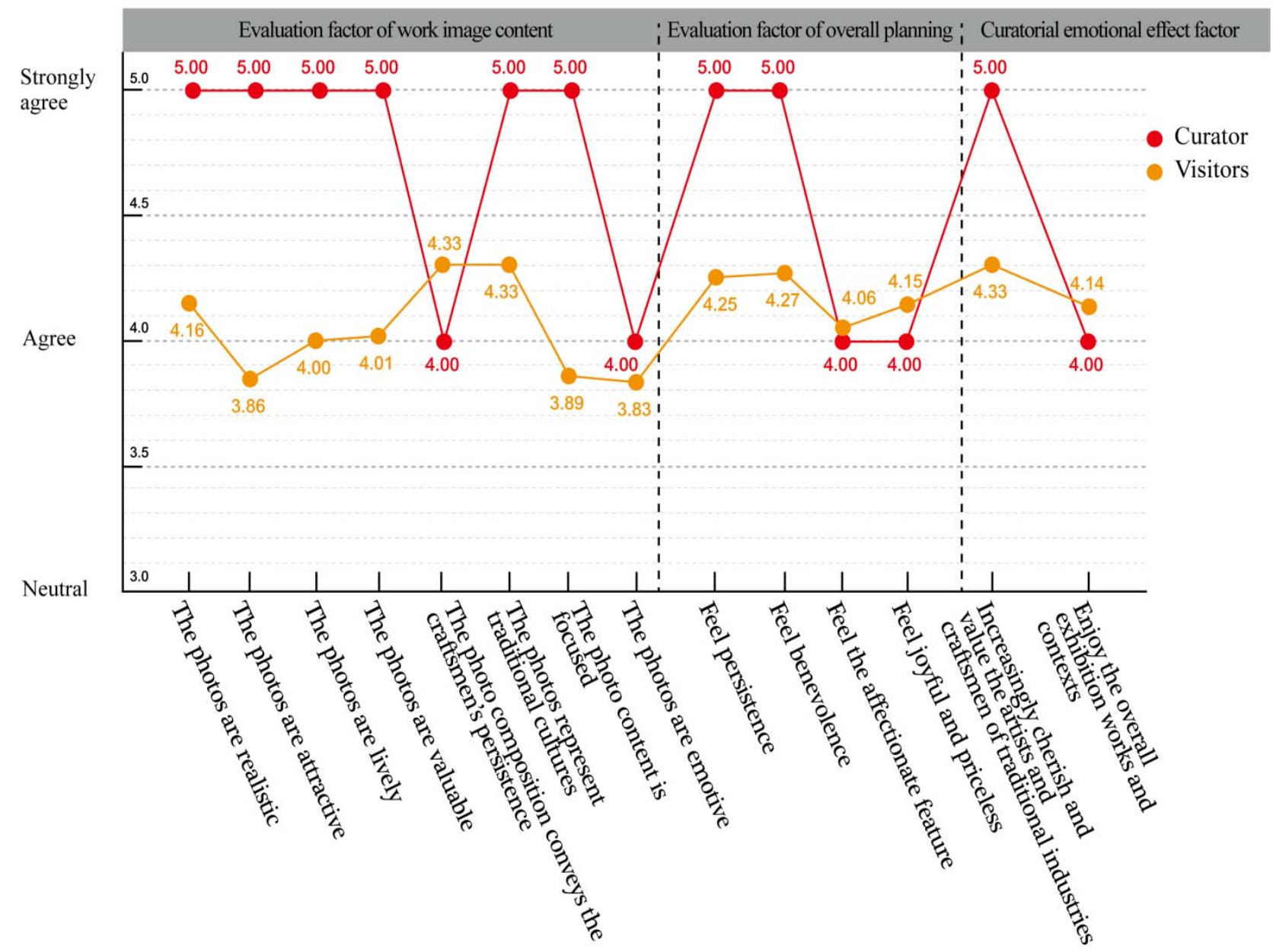

Figure 5. The curator's and visitors' cognitive attitudes and perceived benefits.

In addition to "The photo composition conveys the craftsmen's persistence," the curator rated the other three items (i.e., "I feel the affectionate feature in this exhibition"; "I feel that this exhibition is joyful and priceless"; and "Enjoy the overall exhibition works and contexts") lower than the visitors did. The mean differences were within 0.2 , which could be regarded as the curator's experience being similar to that of the visitors. The smallest difference between curator's and visitors' scores was in "I feel the affectionate feature in this exhibition" from the overall planning evaluation factor. Because this exhibition attempted to evoke the visitors' caring and grateful feelings when examining the artists' and craftsmen's persistence from the exhibits, the curator and the visitors approved of the overall planning. The data reveal that the exhibition successfully and precisely communicated its intended message with the visitors. Through the visitors' cognitive experiences with observing the exhibition and connecting key stimuli that influenced the visitors' emotions, the visitors not only understood the exhibition theme, but also experienced the emotional aspect of the exhibition. 


\section{Conclusion}

The survey results indicate that the curatorial practice model adopted in the curation process can be divided into three stages: (a) project conception; (b) project execution; and (c) project review. The execution stage was further divided into the following six steps: innovative thinking, determining the exhibition theme, establishing the exhibition structure, curating works for the exhibition, designing the venue layout, and reviewing the emotional effect and making improvements. In addition, the following 10-step procedure was employed as the operating method for curating works for the exhibition: 1. Establish the core theme; 2. Establish the thematic connections; 3. Administrative management; 4. Implement engineering technologies; 5. Curate works; 6. Set up the exhibition; 7. Plan the layout of exhibition area; 8. Construct an atmosphere; 9. Evaluate the benefits of the exhibition; and 10. Identify improvement measures. The proposed curatorial practice model was organised on the basis of an actual exhibition implemented by the curator. Through design thinking contexts, execution and creation, as well as review, the model can substantially facilitate curatorial applications for curating agents and organisations.

A curator acts as a mediator. Curators are responsible for facilitating communication and mutual understanding among the host institute, artists, and visitors. Considering how to apply visitor needs and inspire visitors to think and convert them into design elements to successfully curate a fascinating exhibition is critical for every curator. In summary, the quality of an exhibition experience is the main factor influencing the emotional responses of visitors. The findings of this article suggest that the smoothness of visitor traffic flow of the exhibition environment and interactivity between visitors and curate works also influenced the visitors' cognitions and perceptions during their experience. The questionnaire results show that the curator's and visitors' emotional experiences can realise shared conceptual goals. The visitors' preferences and cognitive acceptance were high. Thus, the curatorial practice model can effectively deliver the core values of planning an exhibition concept.

The results of an interview with an experienced curator show that the emotional responses of the visitor based on their experiences have become an indispensable part of curatorial design. In this exhibition, the experienced professional curator transformed the exhibition from merely focusing on exploring disappearing industry sectors and the practice of techniques to creating new visual experiences or questions for a curation theme because the exhibition was implemented by an independent curator leading a team. The curation process was based on a correlation analysis, questionnaire survey, and in-depth interviews, which were used instigate visitor experiences to generate new perspectives, identify emotional values, and market. Consequently, a paradigm of a successful curating case was accomplished, and a curatorial practice model was constructed for future reference. The topic of humanistic representation can provide a reference for exhibition curators seeking to organise events in the future. The model improves the quality and connotation of curations in Taiwan and enables curation to profoundly explore value recreation, thereby, elevating aesthetic literacy in visitor experiences.

\section{References}

Baxter, M. (1998). Product design and development. Taipei: Liuho Publishing House.

Belcher, M. (1991). Exhibitions in museums. Washington, D.C.: Smithsonian Institution Press.

Burcaw, G. E. (1987). Introduction to museum work. Nashville: American Association for State and Local History.

Carroll, A. (2004). Independent curators - A guide for the employment of independent curators. Taipei: Five Senses Arts Management.

Chu, P. Y. (2011). Exploring the emotional dimensions on product design with a decision analysis model (Unpublished Ph.D. dissertation, Graduate Institute of Design Science, Tatung University). 
Crilly, N., Moultrie, J., \& Clarkson, P. J. (2004). Seeing things: Consumer response to the visual domain in product design. Design Studies, 25(6), 547-577.

Falk, J. H., \& Dierking, L. D. (1992). The museum experience. Washington, D.C.: Whalesback Books.

Fu, H. M. (2004). A study on issues deriving from the outsourcing of exhibitions by Taiwan's public museums (Unpublished master's thesis, Graduate Institute of Museology, Tainan National University of the Arts).

Hsu, C. H., \& Lin, R. T. (2011). A study on cultural product design process. Journal of Design, 16(4), 1-18.

Hsu, W. R. (2009). Tendency of international curating and observation symposium: Formation and future trend of curating systems. ARTCO, 203, 12-23.

Jordan, P. W. (2000). Designing pleasurable products. London, U.K.: Taylor \& Francis.

Kao, C. H. (2002). Recreational sports business management. Taoyuan: Wisdom Win Distributor Co..

Lin, P. (2014). Art exhibition: Its value and spatial relationships. Museology Quarterly, 19(1), 29-37.

Lin, R. (2007). Transforming Taiwan aboriginal cultural features into modern product design —A case study of cross cultural product design model. International Journal of Design, 1(2), 47-55.

Liu, S. F. (2003). Research of communication and visitors behaviors in museums—A study of explanatory labels in "The Physical World” Exhibition Room at the National Museum of Natural Science (Unpublished master's thesis, Department of Visual Communication Design, National Yunlin University).

Maslow, A. H. (1943). A theory of human motivation. Psychological Review, 50(4), 370-396.

McLuhan, R. (2000). Go live with a big brand experience. London, U.K.: Haymarket Business Publications.

Norman, D. A. (2005). Emotional design: Why we love (or hate) everyday things. New York, N.Y.: Basic Books.

Oppenheimer, F. (1990). Exhibit conception and design. Museology Quarterly, 4(2), 15-25.

Richins, M. L. (1997). Measuring emotions in the consumption experience. Journal of Consumer Research, 242, 127-146. 